Musées, Patrimoine et Culture scientifiques et techniques

$188 \mid 2020$

mars-avril 2020

\title{
Évaluation d'une application dans le cadre de visites scolaires
}

Inga Walc

\section{OpenEdition}

\section{Journals}

Édition électronique

URL : https://journals.openedition.org/ocim/3656

DOI : 10.4000/ocim.3656

ISSN : 2108-646X

Éditeur

OCIM

Édition imprimée

Date de publication : 1 mars 2020

Pagination : 30-37

ISSN : 0994-1908

Référence électronique

Inga Walc, "Évaluation d'une application dans le cadre de visites scolaires », La Lettre de I'OCIM [En

ligne], 188 | 2020, mis en ligne le 01 mars 2021, consulté le 17 juillet 2021. URL : http://

journals.openedition.org/ocim/3656 ; DOI : https://doi.org/10.4000/ocim.3656

Ce document a été généré automatiquement le 17 juillet 2021.

Tous droits réservés 


\title{
Évaluation d'une application dans le cadre de visites scolaires
}

\author{
Inga Walc
}

Illustration d'après une vue en enfilade au sein de la Maison de Victor Hugoà Paris.

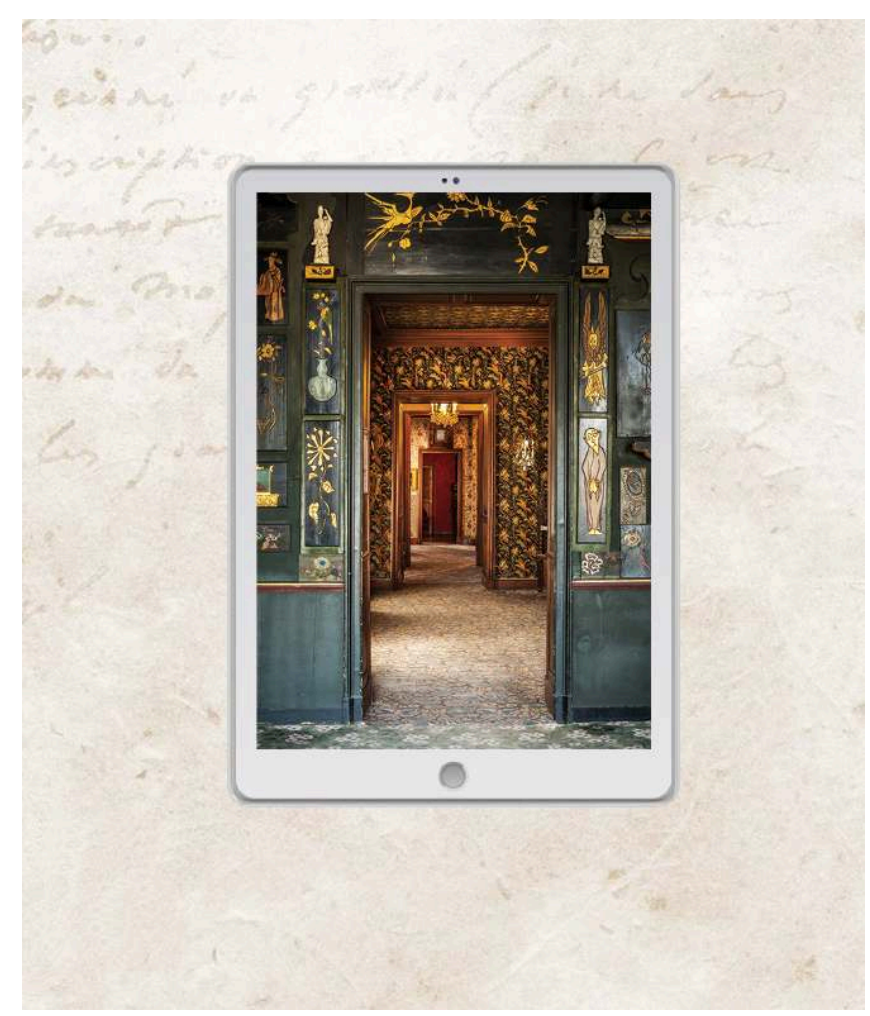

(c) Ocim d'après P. Antoine/Maison de Victor Hugo

L'application Connaître Victor Hugo ${ }^{1}$, utilisée auprès des classes de collège, a fait l'objet d'une étude analysant trois problématiques: les motivations de l'enseignant prescripteur, l'acquisition des connaissances et la satisfaction des élèves. L'étude a été 
menée dans le cadre du master des Approches innovantes de la recherche et de l'enseignement au Centre des recherches interdisciplinaires de l'université Paris Descartes (CRI).

\section{Un musée, deux maisons}

2 Ouverte en 1903, la Maison de Victor Hugo est un des plus anciens musées monographiques français. Aujourd'hui elle accueille des visiteurs dans deux lieux : le musée parisien place des Vosges et la maison d'exil à Guernesey ${ }^{2}$. Les collections, provenant initialement des dons des proches et de la famille $\mathrm{Hugo}^{3}$, ont été enrichies et comportent des fonds de dessins, décor, mobilier, photographies, estampes, manuscrits et correspondance, peintures, sculptures, archives et divers objets. Le mobilier de l'appartement de la place Royale ayant été dispersé avant la création du musée ${ }^{4}$, sa muséographie privilégie le récit de la biographie de l'écrivain à travers des period rooms. Cette approche permet de montrer au public à Paris une partie des décors et du mobilier de la maison de Juliette Drouet à Guernesey ${ }^{5}$ ainsi que les meubles provenant de la dernière chambre de l'écrivain, avenue d'Eylau.

La Maison de Victor Hugo à Paris, dotée d'un service des publics, organise des programmes éducatifs, des événements, des ateliers ou des formations toute l'année. Sa fréquentation dépasse 175000 visiteurs par an, $10 \%$ des visiteurs sont des élèves venant dans le cadre de visites scolaires, les collégiens représentant respectivement un peu plus de $30 \%$ du public scolaire et $3 \%$ du public total. Les classes de l'école primaire constituent $40 \%$ du public scolaire et les centres de loisir $10 \%$ de celui-ci. Les classes de lycée correspondent à $20 \%$ du public scolaire fréquentant le musée.

Alors que Hauteville House - selon l'expression du fils âné de l'écrivain « un autographe sur trois étages » - est considérée comme une œuvre à part entière et sa présentation au public tend vers la restitution de l'état conçu par Hugo, le musée parisien doit composer avec un espace, un imaginaire et un discours muséographique plus complexe.

En effet, à la fois musée littéraire et musée de Beaux-Arts, lieu de vie et espace mémoriel, le musée parisien élabore un récit croisant plusieurs approches.

L'application s'articule autours de deux «Parcours » pour faciliter l'acquisition des connaissances dans et hors du musée. Ici Victor Hugo - décorateur, une activité du " Parcours musée » permet d'appréhender différemment la muséographie.

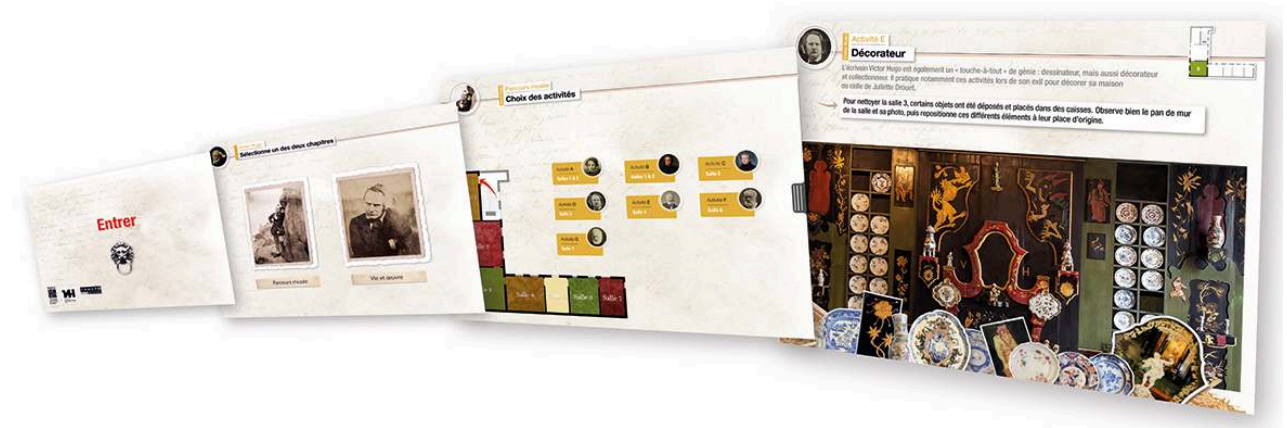

(C) P. Antoine/Maison de Victor Hugo 


\section{Une application pourquoi ? Pour qui ?}

6 La muséographie, constituée par les period rooms offrant des évocations biographiques ou littéraires, est appréciée par le public initié. La lisibilité de cet espace peut s'avérer par contre délicate pour une partie des visiteurs - notamment des jeunes ou des personnes ne possédant pas les connaissances nécessaires. Le choix de création d'un outil numérique s'est porté sur le niveau collège permettant d'encourager une utilisation autonome.

\section{Conception de l'application Connaître Victor Hugo}

7 Le développement et l'intégration ont été réalisés par l'agence Kiupe tandis que la conception des contenus de l'application a été assurée par le service des publics de la Maison de Victor Hugo et le réseau Canopé de Paris ${ }^{6}$. Les contenus de l'application ont été élaborés selon l'importance des œuvres au regard de la biographie de l'écrivain et leur lisibilité pour des collégiens. Enfin, les liens avec les programmes de classes de collège ont été pris en compte, tout comme l'accessibilité de ces œuvres dans l'espace, leur présence constante dans le musée, etc.

8 Les réponses dans chaque activité prennent la forme d'un score graphique permettant de voir l'avancement dans les activités, la proportion des activités réalisées entièrement ou partiellement, de réponses justes et de réponses fausses.

9 Les principales fonctionnalités techniques de l'application sont: des "glisser-déposer " permettant de reconstituer une image, de colorier une frise chronologique, d'associer un bloc d'image à un texte; la sélection de philtres de couleur et d'éclairage d'une œuvre ; des réponses écrites à l'aide du clavier ; la sélection d'un élément de réponse...

\section{Contenus de l'application Connaître Victor Hugo}

Les activités sont organisées en deux ensembles : «Parcours musée » et «Parcours vie et œuvre ». Les sept activités de la partie «Parcours musée » nécessitent la présence dans les salles du musée, alors que les cinq items de «Parcours vie et œuvre » peuvent être réalisés au préalable. L'ensemble des items comportent: un titre, une phrase introductive, une consigne, une "activité ", un module de validation/correction, puis une question de synthèse de la thématique avec le choix entre deux blocs de textes avec, de nouveau, un module de validation/correction.

\section{Le « Parcours musée »}

11 Ce parcours favorise l'observation des œuvres in situ alternant les consignes de recherche, d'identification, de comparaison et d'expérimentation visuelle :

12 - pour répondre aux devinettes visuelles de "À la rencontre de sa famille», il faut retrouver dans les deux premières salles de l'appartement de Victor Hugo les portraits de ses proches;

13 • « Notre-Dame - le roman de la célébrité » comporte l'observation d'œuvres mais aussi de la lecture. Des illustrations du roman sont à associer à de courts textes ; 
14 • «Un buste pour un écrivain » nécessite la mémorisation visuelle de deux sculptures disposées dans deux salles différentes;

15 - dans "Victor Hugo - décorateur ", face aux panneaux décoratifs du salon chinois, les élèves reconstituent l'image du décor ;

16 - lors de l'activité « V. Hugo et ses pièces montées », l'examen des dessins préparatoires et des meubles imaginés par l'écrivain est l'occasion de comprendre son processus créatif;

17 - dans la "Galerie des grands hommes ", un jeu d'association de différentes lumières rend facilement perceptibles la composition et le cadrage d'un célèbre portrait par Bonnat ;

18 - «Je vois de la lumière noire " confronte les élèves avec un portrait mortuaire, proposant d'en relever et identifier la dédicace.

\section{Le «Parcours vie et œuvre»}

19 Ce parcours propose des activités liées à la biographie de Victor Hugo demandant l'observation des images et leur confrontation avec des textes :

20 • dans «Un appartement aménagé en musée » par un jeu de tracé est abordé l'examen des deux plans et de l'évolution de l'espace ;

21 • «Un géant de la littérature » est un simple puzzle qui sous prétexte de rangement de la bibliothèque rappelle les principaux titres d'œuvres de Victor Hugo introduisant la notion du genre littéraire : poésie, théâtre et roman;

22 - les activités «Vie et combats de Victor Hugo » et « De l'engagement politique à l'exil » constituent des synthèses et recourent à des chronologies (à compléter ou colorier et comportant des images ou des textes à classer, ou à associer) ;

23 • «Notre-Dame» est un court exercice d'association d'illustrations à la lecture d'un extrait présentant les personnages du roman.

L'activité Vie et combats de Victor Hugo du « Parcours Vie et œuvre » est un exercice de synthèse des connaissances sur l'auteur.

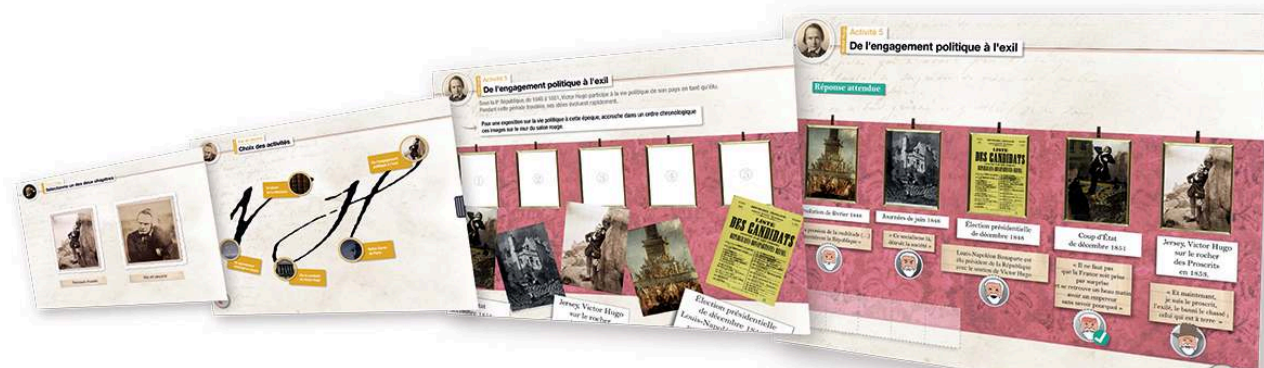

\section{Évaluation en contextede visite scolaire}

Conçue comme une réponse possible à la complexité du discours muséographique de la Maison de Victor Hugo à Paris, difficile à appréhender pour des adolescents, cette 
application leur permet-elle de mieux saisir les principales informations, les retenir, mais aussi de faire l'expérience d'une exploration personnelle? conçue par Roxane Saint-Bauzel ${ }^{7}$, ayant conduit une étude d'évaluation de l'application de visite scolaire Connaitre Leclerc Moulin. En effet, s'appuyant sur ses conclusions, nous avons souhaité comparer la mémorisation, le taux de satisfaction et le comportement lors de trois situations différentes: visite avec application, visite avec conférencier, visite mixte (moitié de temps avec conférencier et moitié avec l'application) ${ }^{8}$.

\section{Typologie des données}

L'analyse se concentre sur la comparaison de la mémorisation des connaissances par les élèves selon le type de visite, ensuite les résultats du questionnaire de satisfaction sont comparés aux résultats quantitatifs d'acquisition des connaissances.

Le questionnaire de satisfaction avait pour objectif de mesurer l'intérêt intrinsèque suscité par l'outil numérique, le taux de satisfaction lors de la visite, la perception par les élèves de leur taux de participation et d'autonomie. La méthodologie de ce questionnaire a été inspirée par les échelles de mesures de motivation et de satisfaction élaborées par Vallerand'.

Le questionnaire adressé aux professeurs visait à rassembler les informations sur leurs attentes générales vis-à-vis d'une visite avec une classe, leurs habitudes à utiliser des outils numériques et le déroulement de la visite.

L'analyse des questionnaires et entretiens avec les adultes encadrant les élèves (professeurs, intervenants culturels, agents d'accueil) tente de formuler des préconisations pour élargir l'offre de médiation muséale proposée aux classes de collégiens. 


\section{Entretiens avec les personnels du musée}

Les conférenciers ont souligné lors des entretiens la difficulté de s'adapter à un nouveau mode de visite. Les agents d'accueil ont dans l'ensemble bien contribué à la bonne marche des visites et de l'étude. Dans des espaces restreints des salles muséales, l'acceptation de l'autonomie des élèves (déplacements, consultation de la documentation, positionnement dans l'espace, etc.) semble être compliquée pour l'institution habituée aux autres usages très codifiés.

Entrée de laMaison deVictor Hugoà Paris.

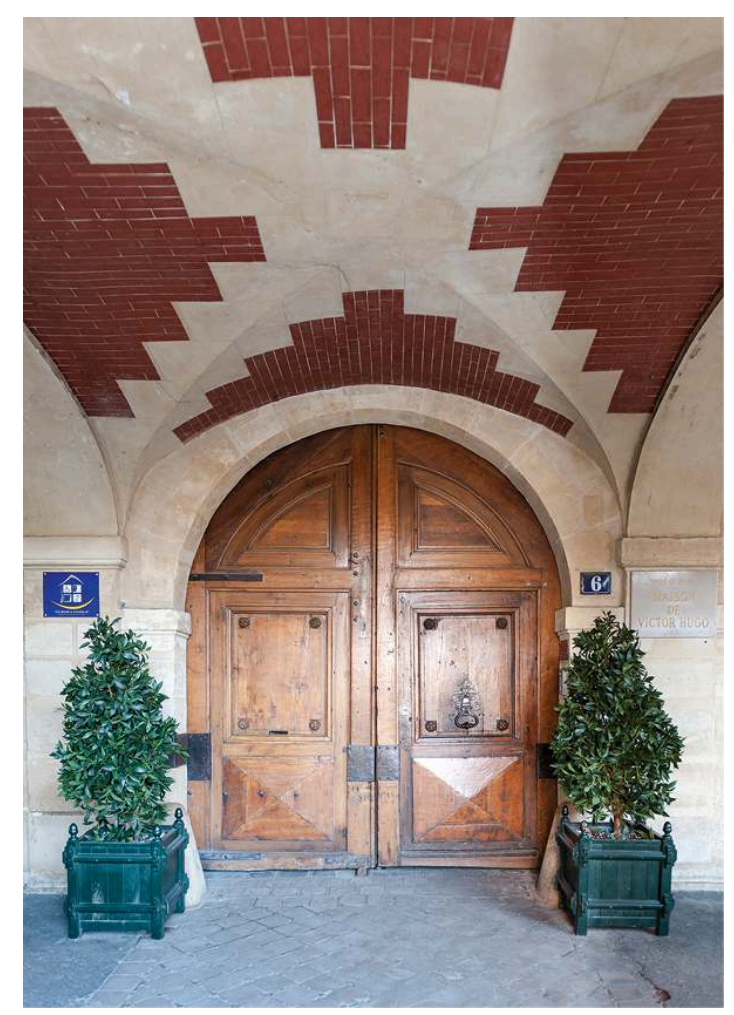

(c) P. Antoine

\section{Résultats de l'expérimentation}

Les élèves ont retenu globalement plus d'informations après la visite avec le conférencier ( $86 \%$ de bonnes réponses) qu'après la visite avec l'application $(66 \%)^{10}$. La comparaison de la proportion de réponses fausses et de réponses manquantes (respectivement: fausses $5 \%$ avec le conférencier et $22 \%$ avec l'application; manquantes: $9 \%$ et $12 \%$ ) pourrait signifier que les élèves utilisant l'application expérimentent et tâtonnent alors que ceux qui ont été exposés à la parole d'un adulte répondent avec plus de certitude.

Le score de bonnes réponses après la visite avec conférencier est de $86 \%$, de $72 \%$ lors de la visite mixte et de $66 \%$ pour les élèves ayant visité le musée avec l'application.

C'est par contre le ressenti des élèves quant à leur appréciation du lieu, la motivation et la satisfaction qui sont différentes des deux autres modes de visite. 


\section{Satisfaction des élèves} encouragées. Par ailleurs, l'outil ne présentant pas de variantes, ou de degrés de difficulté différents, il est jugé «facile » par une partie des élèves, et «compliqué ", 
« lassant » par une autre. Les élèves ont émis le souhait d'une gamification des contenus et des formes des activités.

Le salon rouge de la Maison de Victor Hugo à Paris.

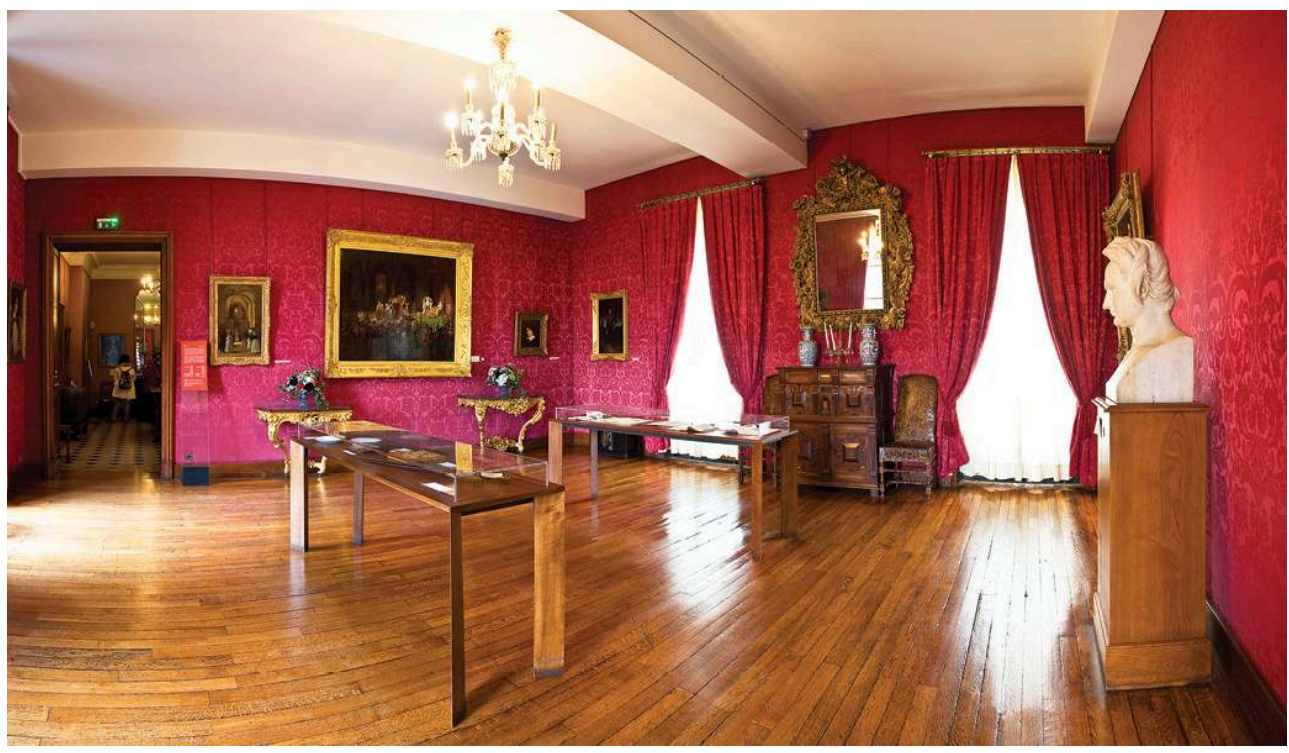

(c) S. Sonnet

\section{Conclusion}

qui concerne la mémorisation des savoirs, nous avons constaté que les élèves retiennent plus d'informations après la visite avec un conférencier, surtout lorsqu'il s'agit des questions liées à la compréhension de grands phénomènes historiques. Le taux de satisfaction des élèves ayant utilisé l'application est cependant plus important que celui des élèves ayant bénéficié de la visite conférence. satisfaction et de l'intérêt pour l'expérience de la visite exprimé par les élèves lors de ce type de visite est nettement supérieur. L'examen plus détaillé des types de réponses erronées, ainsi que des questions non renseignées indique lors de la visite mixte un taux inférieur de « décrochage » de la part des élèves faibles ou moyens.

La conception des outils et activités destinés aux élèves devrait prendre en compte l'ensemble des éléments conditionnant leur motivation et leur autonomie. Tant la combinaison des différents types de médiation, que l'acceptation par l'institution patrimoniale des pratiques culturelles moins codifiées face aux œuvres et à l'espace muséal semblent exercer une influence importante sur la motivation et la satisfaction des jeunes visiteurs. 


\section{NOTES}

1. Description détaillée de l'application Connaître Victor Hugo sur www.maisonsvictorhugo.paris.fr/sites/victorhugo/files/page_simple/documents/ application_connaitre_victor_hugo_dossier_presentation.pdf et téléchagement sur: http:// francaiseapps.fr/iphone-ipad/education/connaitre-victor-hugo-dpogqdq.html

2. En 1927 la demeure de Guernesey a fait l'objet d'une donation par les descendants de V. Hugo à la Ville de Paris, dès lors les deux lieux ont été réunis sous la même direction.

3. Conformément au codicille du testament: «Je donne tous mes manuscrits, et tout ce qui sera trouvé écrit et dessiné par moi, à la bibliothèque nationale de Paris, qui sera un jour la Bibliothèque des États-Unis d'Europe", les manuscrits littéraires et la grande majorité des dessins de l'écrivain se trouvent dans les collections de la BNF. La Maison de Victor Hugo conserve environ 700 dessins de Victor Hugo et un important fonds de lettres provenant de la collection de Paul Meurice et de la famille de l'écrivain.

4. La famille Hugo, contrainte à l'exil, a organisé en juin 1852 une vente des biens et du mobilier.

5. En 1864, Hugo achète à Guernesey une maison au 20 de la rue Hauteville pour Juliette Drouet. Une partie de son décor est présentée dans le parcours de visite du musée parisien.

6. Le réseau Canopé édite des ressources pédagogiques transmédias répondant aux besoins de la communauté éducative :www.reseau-canope.fr ; http://kiupe.com/services

7. Saint-Bauzel R. L'application support de la visite du Musée Moulin-Leclerc, rapport d'expérimentation. Poitiers : Éditions réseau Canopé-DRDUNE, 2014.

8. Les 14 classes de collège ont pu être accueillies avec la répartition suivante : 4 classes pour la visite avec le conférencier ( 82 élèves), 7 classes pour la visite avec l'application (158 élèves) et 3 classes pour la visite mixte (78 élèves).

9. Vallerand R. J., Pelletier L. G., Blais M. R. et Brière N. M., Construction et validation de l'échelle de motivation en éducation (EME), Revue canadienne des sciences du comportement, $n^{\circ} 21$ (3), 1989, pp. 323-349.

Vallerand R. J., Pelletier L. G., Blais M. R., Briere N. M., Senecal C. et Vallieres E. F., The Academic Motivation Scale: A Measure of Intrinsic, Extrinsic, and Amotivation in Education, Educational and Psychological Measurement, $\mathrm{n}^{\circ} 52$ (4), 1992, pp. 1003-1017.

10. Le test statistique (test $t$ de Student) de comparaison entre ces deux groupes donne une différence significative ( $86 \%$ versus $66 \%, \mathrm{p}<0,00001$ ). Dans le cas de visites mixtes - séquence avec conférencier $72 \%$ versus séquence avec application $69 \%-$, cette différence n'est pas significative $(p=0,53)$.

\section{RÉSUMÉS}

La Maison de Victor Hugo à Paris propose une application dédiée à la découverte du musée littéraire. L'auteur revient sur l'expérimentation entreprise auprès d'élèves, afin d'évaluer l'intérêt pédagogique de cet outil de médiation numérique dans le cadre d'une visite scolaire. 
INDEX

Mots-clés : médiation numérique, outil d'aide à la visite, outil pédagogique, public scolaire, centre d'interprétation; musée d'art

\section{AUTEUR}

\section{INGA WALC}

Responsable du Service pédagogique de la Maison de Victor Hugo

inga.walc-bezombes@paris.fr 